\title{
Neurotransmission Regulates Stability of Acetylcholine Receptors at the Neuromuscular Junction
}

\author{
Orlando L. Avila, Daniel B. Drachman, and Alan Pestronk \\ Department of Neurology, Johns Hopkins University, Baltimore, Maryland 21205
}

The majority of acetylcholine receptors (AChRs) at normally innervated neuromuscular junctions are stable, with a halflife averaging about $12 \mathrm{~d}$ in most rodent muscles. Following denervation, the AChRs turn over much more rapidly after a lag period. The mechanism by which motor nerves normally maintain stabilization of junctional AChRs is not yet known. In order to determine whether synaptic transmission plays a role in this process, we have compared the effects of preand postsynaptic chloinergic blockade with those of surgical denervation.

1251- $\alpha$-bungarotoxin was used to label junctional AChRs and follow their loss over time. Presynaptic blockade of quantal ACh transmission was produced in the soleus (SOL) and flexor digitorum brevis muscles of mice by repeated injections of type A botulinum toxin. Postsynaptic blockade of quantal and nonquantal ACh transmission was produced by continuous infusion of $\alpha$-bungarotoxin in the SOL. Our findings show that treatment with botulinum toxin resulted in an accelerated loss of junctional AChRs that was similar to the effects of surgical denervation, though briefly delayed in its onset. Treatment with $\alpha$-bungarotoxin produced an effect that was quantitatively equivalent to the accelerated loss of junctional AChRs following surgical denervation, with an identical time course. These results support the concept that cholinergic synaptic transmission is a mediator of the neural control of stability of junctional AChRs. The possibility that receptor stabilization may represent a mechanism of long-term postsynaptic "memory" dependent on neural transmission is discussed.

Both the distribution and the turnover of acetylcholine receptors (AChRs) of mammalian skeletal muscles are regulated to a large extent by motor nerves (Fambrough, 1979; Drachman et al., 1984; Salpeter and Loring, 1985; Schuetze and Rolc, 1987). In normally innervated mature muscles, $\mathrm{AChRs}$ are present almost exclusively at the postsynaptic membranes of neuromuscular junctions (Miledi, 1960; Albuquerque et al., 1974; Bevan and Steinbach, 1977; Fambrough, 1979; Salpeter, 1987). The majority of junctional AChRs are stable; their half-lives are reported to range from 8 to $13 \mathrm{~d}$ in rodents (Berg and Hall, 1975b;

\footnotetext{
Received Dec. 29. 1988; revised Feb. 22, 1989; accepted Feb. 28.1989.

This work was supported by the US Army, contract DAMD 17-85-C-5069, grants from the Muscular Dystrophy Association and the NIH (IR01 AG07438. 1 R01 NS23719). We are grateful to Dr. D. Ramsay for helpful discussion. to P. Shih for technical assistance, and to $C . F$. Salemi for assistance with the manuscript. Correspondence should be addressed to Daniel B. Drachman, M.D., Department of Neurology. Johns Hopkins University, School of Medicine, $600 \mathrm{~N}$. Wolfe Strect, Baltimore, MD 21205.

Copyright $(1989$ Society for Neuroscience $0270-6474 / 89 / 082902-05 \$ 02.00 / 0$
}

Loring and Salpeter, 1980; Bevan and Steinbach, 1983; Stanley and Drachman, 1983c). There is a growing body of evidence that the stability of these junctional AChRs depends on some function of the motor nerve. (1) Following surgical denervation, the rate of degradation of preexisting stable AChRs accelerates markedly after a lag period of several days, attaining a half-life of 2-5.6 d (Loring and Salpeter, 1980): Levitt and Salpeter, 1981; Stanley and Drachman, 1981; Bevan and Steinbach, 1983). (2) After reinnervation of the denervated muscle, the $A C h R s$ are again stabilized (Salpeter et al., 1986). (3) Recently we have found that the initial stabilization of new AChRs after insertion at normal mature neuromuscular junctions also requires innervation (Stanley and Drachman, 1983a,c; D. Ramsay and D. B. Drachman, unpublished observations). Taken together, this evidence indicates that the motor nerve provides some influence that results in stabilization of junctional AChRs and maintenance of their stability.

The mechanism by which the nerve's receptor-stabilizing influence is mediated is not yet known. Since cholinergic synaptic transmission has been shown to play a role in the neural regulation of other properties of skeletal muscles, including the synthesis and insertion of extrajunctional $\mathrm{AChRs}$, and the resting membrane potential (Thesleff, 1960; Berg and Hall, 1975a; Pestronk et al., 1976; Mathers and Thesleff, 1978; Rubin ct al., 1980; Drachman et al., 1982), we wondered if ACh transmission might also influence the stability of junctional AChRs. Other hypothetical possibilities include physical contact between nerve and muscle membranes (Steinbach, 1974; Anderson and Cohen, 1977: McMahan et al., 1984) or nontransmitter substances released by the nerve (Albuquerque et al., 1972; Cangiano, 1973). In the present study, we have examined the role of ACh transmission in the maintenance of stability of junctional AChRs in 2 muscles of the mouse, the soleus (SOL) and the flexor digitorum brevis (FDB). These 2 muscles were used because their junctional AChRs have different rates of turnover and different time courses of denervation-induced destabilization. We studied the change in turnover of preexisting junctional AChRs following pharmacologic blockade of cholinergic neuromuscular transmission. To do this we used 2 specific neurotoxins, botulinum toxin and $\alpha$-bungarotoxin ( $\alpha$-BuTx). Botulinum toxin selectively blocks the quantal release of $\mathrm{ACh}$ from motor nerve tcrminals, whereas $\alpha$-BuTx blocks AChRs postsynaptically, thereby interrupting both quantal and nonquantal $\mathrm{ACh}$ transmission (Brooks, 1956; Thesleff, 1960; Changeux ct al., 1970; Miledi and Potter, 1971; Lee, 1972; Kao et al., 1976).

Our findings show that treatment with botulinum toxin resulted in an accelerated loss of stable AChRs that was similar to the effect of surgical dencrvation, though delayed in its onset. Treatment with $\alpha$-BuTx quantitatively reproduced the effect of 
surgical denervation, with an identical time course. These results support the concept that cholinergic synaptic transmission plays an important role in mediating the neural control of turnover of stable junctional AChRs. The implication that similar mechanisms of transmitter-driven receptor stabilization may contribute to synaptic "memory" is discussed.

\section{Materials and Methods}

Experimental design. In order to compare the effects of pharmacologic blockade of neuromuscular transmission and denervation on the stability of junctional AChRs, we studied the turnover of junctional AChRs by monitoring the loss over time of ${ }^{123}[-\alpha-B u T x$ that was specifically bound to junctional $A C h R s$ in vivo. The loss of bound ${ }^{125}[-\alpha-B u T x$ has been widely used to follow the loss of AChRs in skeletal muscles (Berg and Hall, 1975b; Devreotes and Fambrough, 1975; Merlie et al., 1976; Bevan and Steinbach, 1977; Gardner and Fambrough, 1979; Loring and Salpeter, 1980; Stanley and Drachman, 1983c, 1987; Salpeter et al., 1986; Ramsay et al., 1988).

In each series of experiments, we first prepared a pure population of labeled stable junctional AChRs, as described below. We then measured (1) the turnover of "control" stable junctional AChRs (i.e., without pharmacologic treatment or denervation); (2) the effect of surgical denervation on the turnover of junctional AChRs; (3) the effect of treatment with botulinum toxin or $\alpha$-BuTx on the turnover of junctional AChRs. We were thus able to compare the effects of denervation and pre- and postsynaptic pharmacologic blockade of $\mathrm{ACh}$ transmission on the loss of stable junctional AChRs.

Female Swiss mice were used throughout these experiments; the animals were anesthetized with chloral hydrate $(0.5 \mathrm{gm} / \mathrm{kg}$ body weight $)$ and ether for all procedures.

Labeling of $A C h R s$ with $125 \% \alpha-B u T x$. AChRs of SOL muscles were labeled by injecting ${ }^{1: 5} \mathrm{I}-\alpha-\mathrm{BuTx}(0.04 \mu \mathrm{g} / \mathrm{gm}$ body weight in $10 \mu \mathrm{lPBS})$ under direct visualization. In the experiment on the FDB muscles, injections were made percutaneously into the sole of the right foot $(0.5$ $\mu \mathrm{g}$ 1251- $\alpha$-BuTx in $20 \mu \mathrm{l}$ PBS). All injections were made via a fine 30 gauge needle.

Preparation of muscles with pure populations of labeled stable AChRs. We have previously found that neuromuscular junctions normally contain 2 populations of AChRs: a subpopulation of rapidly turned over receptors (RTOs) with a half-life of approximately $\mathrm{l} d$ and a majority of stable receptors with a half-life averaging $12 \mathrm{~d}$. In order to follow the turnover of a pure population of stable receptors, we waited $6 \mathrm{~d}$ after labeling the junctional AChRs, so as to allow virtually all the RTOs to be degraded (Stanley and Drachman, 1983c, 1987; Ramsay et al., 1988). At this time point (designated day 0 ), the only remaining labeled AChRs are the stable ones.

Surgical denervation. The SOL and FDB muscles were denervated on day 0 by sectioning the sciatic nerve in the midthigh and avulsing its proximal end to avoid reinnervation.

Presynaptic blockade of synaptic transmission with botulinum toxin. Purified Type A botulinum toxin (generously provided by Dr. E. Schant?) was freshly diluted in mammalian Ringer solution before use. In the SOL muscle, injections were made under direct visualization $(1.5 \times$ $10^{111} \mathrm{gm}$ on day 0 , and $1.0 \times 10^{-111} \mathrm{gm}$ on days 6 and 12). In the FDB muscle, $1.5 \times 10^{-11} \mathrm{gm}$ botulinum toxin was injected percutaneously on day 0 , and $1.0 \times 10^{-10} \mathrm{gm}$ was injected on days 5,12 , and 19 . All control animals received injections of Ringer solution at the same times. A volume of $10 \mu \mathrm{l}$ was used for all injections.

Postsynaptic blockade of $A C h$ transmission with $\alpha-B u T x$. Continuous neuromuscular blockade was produced by perfusing soleus muscles with purified $\alpha$-BuTx via implantable osmotic pumps as previously described (Pestronk et al., 1980; Drachman et al., 1982; Pestronk and Drachman, 1985). Neuromuscular blockade was initiated at day 0 by injecting 1.1 $\mu \mathrm{g} \alpha$-BuTx in PBS directly into the muscle via a fine 30 gauge needle. Blockade was then maintained by continuous perfusion of $\alpha$-BuTx in PBS $(0.06 \mu \mathrm{g} / \mu \mathrm{l}$ at $0.46 \mu \mathrm{l} / \mathrm{hr})$ by means of Alzet model 2002 miniosmotic infusion pumps (Alza Corp., Palo Alto, CA). The pumps were implanted subcutaneously into the back, and the solution was delivered directly over the belly of the SOL via tapered PE60 polyethylene tubing (Clay Adams, Parsippany, NJ) sutured in place. Control animals were injected and perfused in the same manner with similar volumes of PBS. The number of infusion pumps that could be implanted in a single experiment was limited, and we therefore elected to study the 2 time points, i.e., 4 and $6 \mathrm{~d}$, which were found to be critical in our denervation and botulinum experiments. After 4 and $6 \mathrm{~d}$ of treatment, the muscles were removed and the loss of stable junctional AChRs was evaluated as described below (sce Results).

Measurement of turnover of stable junctional AChRs. A baseline was established by removing muscles with labeled stable AChRs from the control group on day 0 . Samples of 4-13 muscles were removed from the control and experimental groups at various times thereafter. Radioactivity bound to junctional AChRs was measured by counting the whole excised muscle in a gamma spectrometer (Micromedic Systems, Inc., Horsham, PA). The counts for each muscle were corrected for decay and then expressed as a fraction of the mean counts of the baseline (day 0) control group for that batch of experimental mice.

In these experiments, ${ }^{125} \mathrm{I}-\alpha$-BuTx bound to the whole SOL or FDB was used as a measure of radioactivity bound to the junctional AChRs. We conducted experiments that showed that at $6 \mathrm{~d}$ or more after labeling, nonspecific or extrajunctional binding was negligible. To assess the degrce of nonspecific binding, we washed the muscles extensively (18 washes over $18 \mathrm{hr}$ ). This removed less than $1 \%$ of the bound radioactivity (unpublished results). In order to assess the degree of extrajunctional binding, we measured the radioactivity in the junctional region relative to extrajunctional regions. We found that $6 \mathrm{~d}$ after labeling more than $98 \%$ of the total muscle radioactivity was bound at the junctional regions (unpublished observations). Thus, whole muscle radioactivity can be used as an accurate measure of junctional radioactivity.

Analysis of data. The data for each muscle (SOL or FDB) at each time point were pooled, means and SEM were calculated, and in the experiments with sufficient numbers of time points, plotted on a semilog graph by the least-squares method. Data for groups of muscles were compared by the Student's 2-tailed $t$ test.

\section{Results}

The results of this study show that treatment of the mouse SOL and FDB muscles with botulinum toxin produced a significant denervation-like accelcration of degradation of labeled stable junctional AChRs, although the onset of this effect was delayed compared with that of surgical denervation. Postsynaptic blockade with $\alpha$-BuTx, which blocks both quantal and nonquantal ACh transmission, produced an effect that was quantitatively equivalent to that of denervation, with an identical time course.

\section{Presynaptic blockade of neuromuscular transmission with botulinum toxin}

Figure 1 shows the degradation curves for stable junctional receptors of the SOL muscle. In the control muscles, bound radioactivity was lost with a half-life of approximately $11.5 \mathrm{~d}$. Within $4 \mathrm{~d}$ after denervation, there was a significantly greater loss of junctional AChRs compared with the controls $(p<0.01)$, and an increase in the rate of degradation with a half-life of 3.6 d. Following treatment with botulinum toxin, the loss of AChRs first became significantly different from controls at $6 \mathrm{~d}$ (i.e., 2 $\mathrm{d}$ later than in the denervated group; $p<0.01)$. The rate of degradation increased, after botulinum treatment, to a half-life of $3.6 \mathrm{~d}$. However, at the $6 \mathrm{~d}$ time point, the loss of labeled junctional $A C h R s$ in botulinum-treated muscles was not as great as that of the denervated muscles at $6 \mathrm{~d}(p<0.01)$.

Figure 2 shows the degradation curves for stable junctional receptors of the FDB muscle. In the control muscles, radioactivity was lost with a half-life of $16.2 \mathrm{~d}$. This rate of degradation was consistently slower than that of the junctional AChRs of the SOL muscle measured here and of the reported rate for the sternomastoid muscles (Loring and Salpeter, 1980; Salpeter et al., 1986; Ramsay et al., 1988). Following denervation, there was a trend toward more rapid degradation of AChRs by day 16 , but the difference between dencrvated and control muscles first became statistically significant at day $20(p<0.001)$. The half-life of junctional AChRs at this time was $4.5 \mathrm{~d}$. Botulinum- 


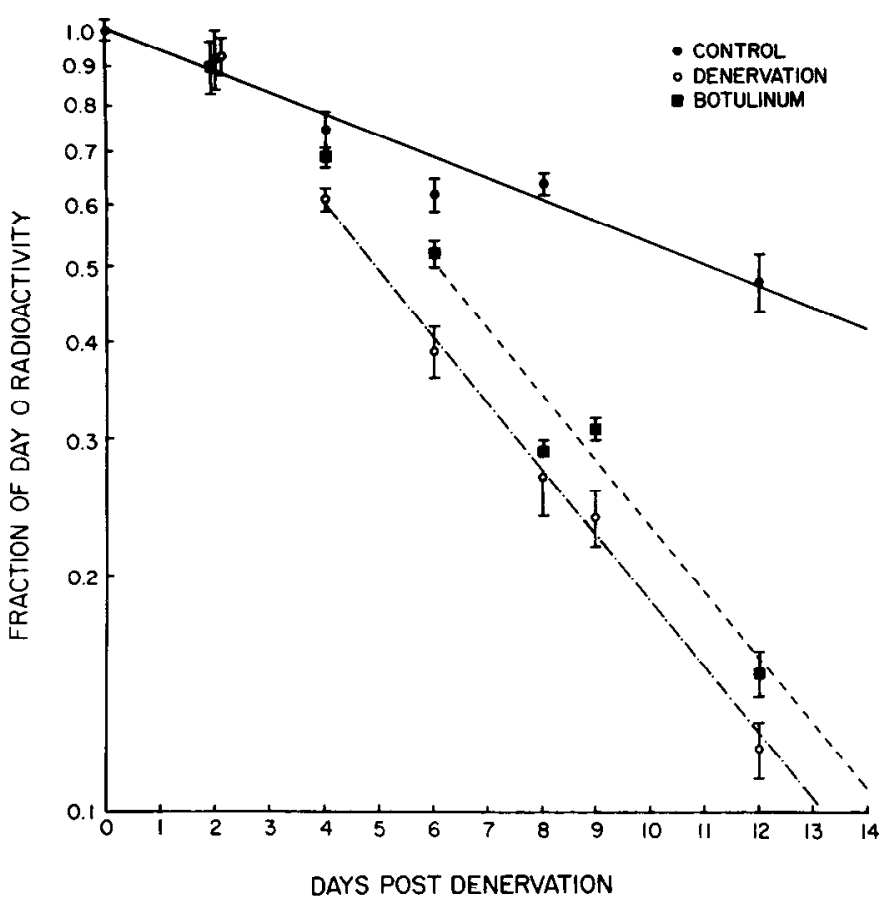

Figure 1. Effect of surgical denervation and botulinum toxin treatment on turnover of stable junctional AChRs in the soleus muscle. SOL muscles of mice were either denervated, treated with botulinum toxin, or treated with control injections as described. Note the accelerated loss of junctional AChRs in the denervated and botulinum-treated muscles. The difference between control and denervated muscles was significant at day $4(p<0.01)$; for botulinum-treated muscles, the difference first became significant at day $6(p<0.01)$.

treated muscles also showed a greater loss of labeled junctional AChRs, which first reached statistical significance later, at day $29(p<0.01)$.

As noted above, the accelerated turnover of AChRs began earlier after denervation than after botulinum treatment; in the SOL muscle, the loss of stable junctional AChRs was significantly greater at 4 and $6 \mathrm{~d}(p<0.01)$.

\section{Postsynaptic blockade of ACh transmission with $\alpha-B u T x$}

In order to compare the effects of postsynaptic blockade of $\mathrm{ACh}$ transmission with those of denervation, we measured junctional AChRs of SOL muscles at 4 and $6 \mathrm{~d}$ after the beginning of $\alpha$-BuTx treatment and denervation. Table 1 shows that both procedures produced a highly significant loss of junctional AChRs at 4 and $6 \mathrm{~d}$, as compared with controls $(p<0.01)$. Most im-

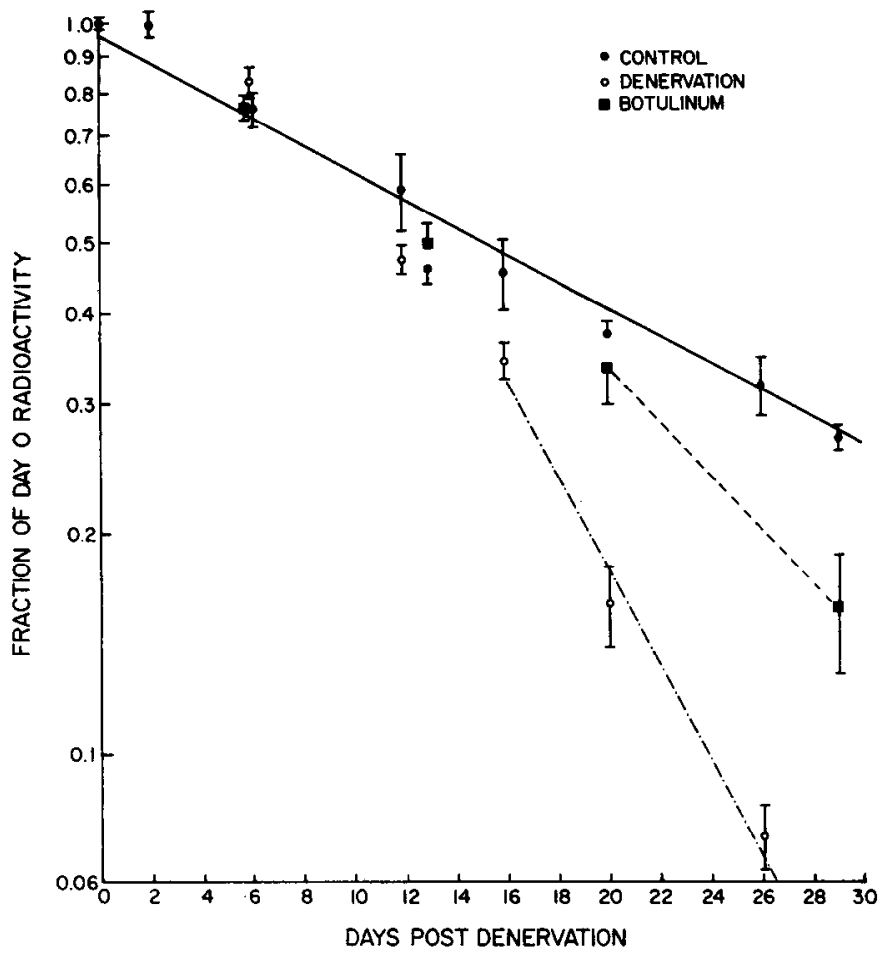

Figure 2. Effect of surgical denervation and botulinum toxin treatment on turnover of stable junctional AChRs in the flexor digitorum brevis muscle. Flexor digitorum brevis muscles of mice were treated as in Figure 1. Note the accelerated loss of junctional AChRs in the denervated muscles, reaching significance at day 20 and in the botulinumtreated muscles at day 29.

portant, the effects of $\alpha$-BuTx treatment did not differ from those of denervation at either time point $(p>0.1)$.

\section{Discussion}

The present investigation was designed to examine the role of neuromuscular synaptic transmission in the maintenance of stability of junctional AChRs. Treatment with botulinum toxin, which blocks neuromuscular transmission presynaptically, and $\alpha$-BuTx, which acts postsynaptically, resulted in "destabilization" of preexisting AChRs at neuromuscular junctions, similar to the effect of denervation. We first compared the effects of botulinum treatment and denervation in two muscles, the SOL and the FDB. The normal rates of degradation of junctional AChRs and the time course of denervation-induced destabilization are consistently different in these 2 muscles. In both

Table 1. Rapid loss of junctional AChRs following $\alpha$-BuTx treatment or denervation

\begin{tabular}{lll} 
& Experimental period & \\
\cline { 2 - 2 } Treatment & $4 \mathrm{~d}$ & $6 \mathrm{~d}$ \\
\hline Ringer's infusion (controls) & $0.84 \pm 0.03(n=6)$ & $0.70 \pm 0.05(n=5)$ \\
$\alpha$-Bungarotoxin infusion & $0.59 \pm 0.04(n=7)^{a . h}$ & $0.48 \pm 0.03(n=11)^{a . h}$ \\
Denervation & $0.64 \pm 0.02(n=7)^{a}$ & $0.46 \pm 0.02(n=6)^{a}$
\end{tabular}

The proportion of junctional AChRs remaining at each time point is expressed as the mean fraction of the day 0 control values \pm SEM. Note the loss of junctional AChRs in $\alpha$-BuTx-treated and denervated muscles as compared with Ringer's controls. $n=$ number of animals.

${ }^{a}$ Less than control $p<0.01$.

${ }^{b}$ Not different from denervation, $p>0.1$. 
muscles, botulinum toxin treatment resulted in an unequivocal denervation-like acceleration of the turnover of junctional AChRs. However, in both muscles, the onset of the effect of botulinum began later than that of dencrvation. By contrast, postsynaptic blockade using $\alpha$-BuTx quantitatively reproduced the effects of denervation on the destabilization of junctional AChRs, with an identical time course.

The interpretation of these results depends on an understanding of the actions of the 2 neurotoxins used. Botulinum toxin is a specific presynaptic inhibitor of the quantal release of $\mathrm{ACh}$ (both impulse-dependent and spontaneous) from cholinergic nerves (Brooks, 1956; Thesleff, 1960; Kao et al., 1976; Simpson, 1981; Stanley and Drachman, 1983b). It does not block the nonquantal release of ACh (Stanley and Drachman, 1983b) and has no other known actions on nerves or muscles. In particular, it does not produce structural damage (Thesleff, 1960) nor does it interfere with axonal transport (Pestronk et al., 1976). In contrast, the action of $\alpha$-BuTx is postsynaptic; numerous studies have demonstrated the specificity of action of $\alpha$-BuTx in blocking the ligand-binding sites of nicotinic junctional AChRs (Changeux et al., 1970; Miledi and Potter, 1971; Lee, 1972). $\alpha$-BuTx is therefore strategically located to interfere with both quantal and nonquantal $\mathrm{ACh}$ transmission, in contrast to the quantal blockade produced by botulinum toxin. Although the binding of $\alpha$-BuTx to AChRs is virtually irreversible, the rapid synthesis and insertion of new AChRs at neuromuscular junctions (Ramsay et al., 1988) requires that $\alpha$-BuTx be applied continuously to maintain persistent blockade of ACh transmission. Prolonged application of $\alpha$-BuTx by the perfusion method used here does not damage neuromuscular junctions morphologically or interfere with fast axonal transport (Drachman et al., 1982). By virtue of its specificity, it is highly improbable that $\alpha$-BuTx could interfere with the transport, delivery, or action of hypothetical "trophic" agents other than ACh.

The only known common denominator of action of botulinum toxin and $\alpha$-BuTx is interference with ACh transmission. Therefore, the most straightforward interpretation of our findings is that blockade of ACh transmission is responsible for the denervation-like destabilization of junctional AChRs. This suggests that neuromuscular transmission and its consequences (which include muscle contraction) play a major, if not exclusive, role in mediating the motor nerve's effect on stability of junctional AChRs.

The delayed onset of increased AChR turnover after botulinum treatment, as compared with denervation or $\alpha$-BuTx treatment, was a consistent finding in our experiments. This cannot be attributed to a delay in the cholinergic blocking action of botulinum toxin since complete paralysis and nearly complete cessation of miniature endplate potentials occur within a few hours after injection of botulinum toxin (Kao et al., 1976; Pestronk et al., 1976). An alternative possibility is that the failure of botulinum toxin to block the spontaneous nonquantal release of ACh (Stanley and Drachman, 1983b) may account for the difference between its effects and those of denervation or $\alpha$-BuTx treatment on the destabilization of junctional AChRs. This is consistent with previous observations that nonquantal $\mathrm{ACh}$ transmission has a significant partial influence in regulating certain other properties of muscles (Mathers and Thesleff, 1978; Drachman et al., 1982).

The mechanism by which ACh transmission influences the stability of junctional AChRs is not yet known. Clearly, the effects of ACh cannot be exerted directly on the AChRs, whose turnover is being followed in these experiments, since the ${ }^{125} \mathrm{I}$ $\alpha$-BuTx used as a label itself blocks the ligand-binding sites of these AChRs. However, ACh transmission acting on nonblocked AChRs at the same junctions presumably is responsible for mediating a stabilizing effect. Although the differences between stable and destabilized AChRs have as yet been defined only in terms of their turnover times, this undoubtedly reflects some biochemical or structural differences between them. Possible differences include covalent modifications of the AChR molecules (for example, by phosphorylation, acylation, or methylation) (reviewed by Salpeter and Loring, 1985), attachment of AChRs to cytoskeletal elements (Bloch and Hall, 1983; Froehner, 1986), or alterations in the surrounding microenvironment of the synaptic membrane (McMahan et al., 1984).

Perhaps the most important implication of the present work is its possible relation to synaptic memory processes. Previous studies of synaptic memory have focused for the most part on presynaptic mechanisms that modify neurotransmission (Kandel et al., 1987). By contrast, stabilization of AChRs constitutes a long-term modification of the neuromuscular junction at a postsynaptic level. AChR stabilization takes place over a much more extended time scale than the phenomenon of receptor "desensitization," which has previously been proposed as a form of short-term postsynaptic memory (Changeux et al., 1984, 1987). Thus, AChR stabilization represents a model of long-term postsynaptic "memory," albeit at a peripheral synapse between motor nerve and skeletal muscle cells. The present results demonstrate that neurotransmission plays a key role in mediating this process. We suggest that similar mechanisms of transmitterdriven receptor stabiization may be involved in memory processes in the CNS as well.

\section{References}

Albuquerque, E. X., J. E. Warnick, J. R. Tasse, and F. M. Sansone (1972) Effects of vinblastine and colchicine on neural regulation of fast and slow skeletal muscles of the rat, Exp. Neurol. 37:607-634.

Albuquerque, E. X., E. A. Barnard, C. W. Porter, and J. E. Warnick (1974) The density of acetylcholine receptors and their sensitivity in the post synaptic membranes of muscle endplates. Proc. Natl. Acad. Sci. USA 71: 2818-2822.

Anderson, M. J., and M. W. Cohen (1977) Nerve induced and spontaneous redistribution of acetylcholine receptors of cultured muscle cells. J. Physiol. (Lond.) 268: 757-773.

Berg, D. K., and Z. W. Hall (1975a) Increased extrajunctional acetylcholine sensitivity produced by chronic post-synaptic neuromuscular blockade. J. Physiol, (Lond.) 244: 659-679.

Berg, D. K., and Z. W. Hall (1975b) Loss of $\alpha$-bungarotoxin from junctional and extrajunctional acetylcholine receptors in rat diaphragm muscle in vivo and in organ culture. J. Physiol. (Lond.) 252: 771-789.

Bevan, S., and J. H. Steinbach (1977) The distribution of $\alpha$-bungarotoxin binding sites of mammalian skeletal muscle developing in vitro. J. Physiol. (Lond.) 267: 195-213.

Bevan, S., and J. H. Steinbach (1983) Denervation increases the degradation rate of acetylcholine receptors at end plates in vivo and in vitro. J. Physiol. (Lond.) 336: 159-177.

Bloch, R. J., and Z. W. Hall (1983) Cytoskeletal components of the vertebrate neuromuscular junction: vinculin, $\alpha$-actinin, and filamin. J. Cell Biol. 97: 217-223.

Brooks, V. (1956) An intracellular study of the action of repetitive nerve volleys and of botulinum toxin on miniature endplate potentials. J. Physiol. (Lond.) 134: 264-277.

Cangiano, A. (1973) Acetylcholine supersensitivity: The role of neurotrophic factors. Brain Res. 58: 255-259.

Changeux, J.-P., M. Kasai, and C.-Y. Lee (1970) Use of a snake venom to characterize the cholinergic receptor protein. Proc. Natl. Acad. Sci. USA 67: 1242-1247. 
Changeux, J.-P., A. Devillers-Thiery, and P. Chemouilli (1984) Acetylcholine receptor: An allosteric protein. Science 225: 1335-1345.

Changeux, J.-P., A. Klarsfeld, and T. Heidmann (1987) The acetylcholine receptor and molecular models or short- and long-term learning. In The Neural and Molecular Basis of Learning, J.-P. Changeux and M. Konishi, eds., pp. 31-84, Wiley, New York.

Devreotes, P. M., and D. M. Fambrough (1975) Acetylcholine receptor turnover in developing muscle fibers. J. Cell Biol. 65: 335-358.

Drachman, D. B., E. F. Stanley, A. Pestronk, J. W. Griffin, and D. L. Price (1982) Neurotrophic regulation of two properties of skeletal muscle by impulse-dependent and spontaneous acetylcholine transmission. J. Neursci. 2: 232-243.

Drachman, D. B., E. F. Stanley, and A. Pestronk (1984) Neural regulation of muscle properties. In Neuromuscular Diseases, G. Serratrice et al., eds., pp. 415-424, Raven, New York.

Fambrough, D. M. (1979) Control of acctylcholine receptors in skeletal muscle. Physiol. Rev. 59: 165-227.

Froehner, S. C. (1986) The role of the postsynaptic cytoskeleton in AChR organization. Trends Neurosci. 9: 37-41.

Gardner, J. M., and D. M. Fambrough (1979) Acetylcholine receptor degradation measured by density labeling: Effects of cholinergic ligands and evidence against recycling. Cell 16:661-674.

Kandel, E. R., V. F. Castellucci, P. Goelet, and S. Schacher (1987) Cell-biological interrelationships between short-term and long-term memory. In Molecular Neurobiology in Neurology and Psychiarry, E. Kandel ed., pp. 111-132. Raven, New York.

Kao, I., D. B. Drachman, and D. L. Price (1976) Botulinum toxin: Mechanism of presynaptic blockade. Science 193: 1256-1258.

Lee, C. Y. (1972) Chemistry and pharmacology of polypeptide toxins in snake venoms. Annu. Rev. Pharmacol. 12: 265-286.

Levitt, T. A., and M. M. Salpeter (1981) Denervated endplates have a dual population of junctional acetylcholine receptors. Nature 291 : $239-241$

Loring, R. H., and M. M. Salpeter (1980) Denervation increases turnover rate of junctional acetylcholine receptors. Proc. Natl. Acad. Sci. USA 77: 2293-2297.

Mathers, D. A., and S. Thesleff (1978) Studies on ncurotrophic regulation of murine skeletal muscle. J. Physiol. (Lond.) 282: 105-114.

McMahan, U.J., C. R. Slater, and R. M. Marshall (1984) The influence of basal lamina on the accumulation of acetylcholine receptors at synaptic sites in regenerating muscle. J. Cell Biol. 98: 1453-1473.

Merlie, J. P., J.-P. Changeux, and F. Gros (1976) Acetylcholine receptor degradation measured by pulse-chase labeling. Nature 264 : 74-76.

Milcdi, R. (1960) The acetylcholine sensitivity of frog muscle fibres after complete or partial denervation. J. Physiol. (Lond.) 151: 1-23.

Miledi, R., and L. T. Potter (1971) Acetylcholine receptors in muscle fibers. Nature 233: 599-603.

Pestronk, A., and D. B. Drachman (1985) Motor nerve terminal out- growth and acetylcholine receptors: Inhibition of terminal outgrowth by $\alpha$-bungarotoxin and anti-acetylcholine receptor antibodies. J. Neurosci. $5: 751-758$.

Pestronk, A., D. B. Drachman, and J. W. Griffin (1976) Effect of botulinum toxin on trophic regulation of acetylcholine receptors. $\mathrm{Na}$ ture 264: 787-789.

Pestronk, A., D. B. Drachman, E. F. Stanley, D. L. Price, and J. W. Griffin (1980) Cholinergic transmission regulates extrajunctional acetylcholine receptors. Exp. Neurol. 70: 690-696.

Ramsay, D., D. B. Drachman, and A. Pestronk (1988) Rapid synthesis of acetylcholine receptors at neuromuscular junction. Brain Res. 462 . 134-141.

Rubin, L. L., S. M. Schuetze, C. L. Weill, and G. D. Fischbach (1980) Regulation of acetylcholinesterase appearance at neuromuscular junctions in vitro. Nature 283: 264-267.

Salpeter, M. M. (1987) Development and neural control of the neuromuscular junction and of the junctional acetylcholine receptor. In The Vertebrate Neuromuscular Junction, M. M. Salpeter, ed., pp. 55115, Liss, New York

Salpeter, M. M., and R. H. Loring (1985) Nicotinic acetylcholine receptors in vertebrate skeletal muscle: Properties, distribution, and neural control. Prog. Neurobiol. (NY) 25: 297-325.

Salpeter, M. M., D. L. Cooper, and T. Levitt-Gilmour (1986) Degradation rates of acetylcholine receptors can be modified in the postjunctional plasma membrane of the vertebrate neuromuscular junction. J. Cell Biol. 103: 1399-1403.

Schuetze, S. M., and L. W. Role (1987) Developmental regulation of nicotinic actylcholine receptors. Annu. Rev. Neurosci. 10: 403-457.

Simpson, L. L. (1981) The origin, structure, and pharmacological activity of botulinum toxin. Pharmacol. Rev. 33: 155-188.

Stanley, E. F., and D. B. Drachman (1981) Denervation accelerates the degradation of junctional acetylcholine receptors. Exp. Neurol. 73: $390-396$

Stanley, E. F., and D. B. Drachman (1983a) Evidence for the postinsertional stabilization of $\mathrm{ACh}$ receptors at the neuromuscular junction. Soc. Neurosci. Abstr. 9: 844.

Stanley, E. F., and D. B. Drachman (1983b) Botulinum toxin blocks quantal but not non-quantal release of $\mathrm{ACh}$ at the neuromuscular junction. Brain Res. 261: 172-175.

Stanley, E. F. and D. B. Drachman (1983c) Rapid degradation of "new" acetylcholine receptors at neuromuscular junctions. Science 222: 67-69.

Stanley, E. F., and D. B. Drachman (1987) Stabilization of acetylcholine receptors at the neuromuscular junction: Analysis by specific antibodies. Ann. NY Acad. Sci. 505: 121-132.

Steinbach, E. H. (1974) Role of muscle activity in nerve-muscle interactions in vitro. Nature 248: 70-71.

Thesleff, S. (1960) Supersensitivity of skeletal muscle produced by botulinum toxin. J. Physiol. (Lond.) 151: 598-607. 